University of Nebraska - Lincoln

DigitalCommons@University of Nebraska - Lincoln

Mechanical \& Materials Engineering Faculty

Publications

Mechanical \& Materials Engineering,

Department of

2010

\title{
Effects of a Mass Layer With Gradually Varying Thickness on a Quartz Crystal Microbalance
}

\author{
Nan Liu \\ University of Nebraska-Lincoln, nliu1@unl.edu \\ Jiashi Yang \\ University of Nebraska-Lincoln, jyang1@unl.edu \\ Weiqiu Chen \\ Zhejiang University, chenwq@zju.edu.cn
}

Follow this and additional works at: https://digitalcommons.unl.edu/mechengfacpub

Part of the Mechanical Engineering Commons

Liu, Nan; Yang, Jiashi; and Chen, Weiqiu, "Effects of a Mass Layer With Gradually Varying Thickness on a Quartz Crystal Microbalance" (2010). Mechanical \& Materials Engineering Faculty Publications. 70. https://digitalcommons.unl.edu/mechengfacpub/70

This Article is brought to you for free and open access by the Mechanical \& Materials Engineering, Department of at DigitalCommons@University of Nebraska - Lincoln. It has been accepted for inclusion in Mechanical \& Materials Engineering Faculty Publications by an authorized administrator of DigitalCommons@University of Nebraska Lincoln. 


\title{
Effects of a Mass Layer With Gradually Varying Thickness on a Quartz Crystal Microbalance
}

\author{
Nan Liu, Jiashi Yang, and Weiqiu Chen
}

\begin{abstract}
We study the effects of the nonuniform thickness of a thin mass layer on a quartz crystal microbalance. A theoretical analysis is performed on thickness-shear vibration of an AT-cut quartz plate with a nonuniform mass layer. Mindlin's two-dimensional equation for thickness-shear vibration of a quartz plate is used. Free vibration frequencies and modes are obtained. The effects of the mass layer nonuniformity are examined. It is shown that resonant frequencies and energy trapping of thickness-shear modes are sensitive to mass layer nonuniformity.
\end{abstract}

Index Terms-Mass sensor, plate, resonator, thickness shear.

\section{INTRODUCTION}

$\mathbf{Q}$ UARTZ CRYSTAL RESONATORS (QCRs) operating with plate thickness-shear (TSh) modes have been used for monitoring thin-film deposition and mass sensing based on the inertial effect of the thin film or mass layer on the resonant frequencies of the QCR, and are called quartz crystal microbalances (QCMs) for these applications. The effects of a thin film on a QCR are multifold, including the mass density, elastic constants (stiffness), and thickness, etc., of the thin film. In the simplest description (the well-known Sauerbrey equation), the inertia of a thin film lowers the resonant frequencies of a QCR [1], [2]. This effect alone can be used to measure the product of the thin-film mass density and thickness. Researchers also developed more sophisticated one-dimensional models based on pure TSh modes depending on the plate thickness coordinate only, showing both the inertial and stiffness effects of the mass layer [3], [4]. These thickness-mode models are valid for uniform films on unbounded plates only, without in-plane variations or boundary effects. Behaviors of real devices are more complicated for several reasons and can deviate, sometimes considerably, from the results predicted by thickness-mode models. For example, due to the so-called energy-trapping phenomenon, the TSh vibration is not uniform and is mainly under the mass layer and decays exponentially away from the mass layer edge. The in-plane variations of the

Manuscript received November 22, 2010; revised December 20, 2010; accepted December 22, 2010. Date of publication December 30, 2010; date of current version May 25, 2011. This work was supported in part by the National Science Foundation of China (NSFC) under Grant 11090333 and Grant 10725210 and the National Basic Research Program of China under Grant 2009CB623200. The associate editor coordinating the review of this paper and approving it for publication was Prof. Bernhard Jakoby.

N. Liu and J. Yang are with the Department of Engineering Mechanics, University of Nebraska, Lincoln, NE 68588-0526 USA.

W. Chen is with the Department of Engineering Mechanics, Zhejiang University, Hangzhou 310027, China (e-mail: chenwq@zju.edu.cn).

Color versions of one or more of the figures in this paper are available online at http://ieeexplore.ieee.org.

Digital Object Identifier 10.1109/JSEN.2010.2103360

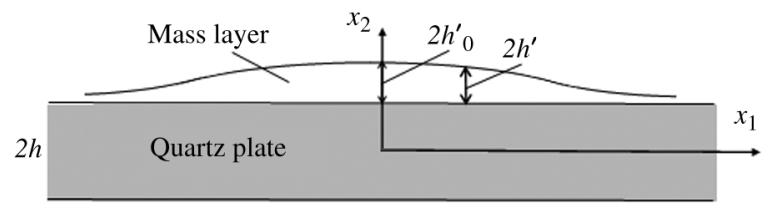

Fig. 1. A quartz plate with a nonuniform mass layer.

TSh modes also cause deviation from the Sauerbrey equation. There have been a few attempts considering the in-plane variations of TSh modes [5]-[7], but overall theoretical results are few and scattered.

Recently, it has been pointed out that the mass layer on a QCM is sometimes nonuniform and little is known about its implications [8]. This is what the present paper is concerned with. While there have been a few published results on nonuniform mass layers or nonuniform electrodes on QCRs either for mass sensing [9], [10] or for energy trapping in resonator applications [11]-[14], these analyses are all for strip resonators with modes and mass layer (or electrode) thickness variations depending on one in-plane coordinate only. For an accurate understanding of the effects of nonuniform mass layers, a more sophisticated analysis with the mass layer thickness and mode variation depending on both of the in-plane coordinates is necessary. In our previous paper [15], a mass layer with a piecewise constant thickness on a QCR was analyzed. The results of [15] showed that the variation of the mass layer thickness has significant effects on the frequencies and mode shapes of the QCR, and further study is necessary. In this paper, we study the case of a mass layer with a continuously varying thickness on a QCR, which is a more realistic situation than the stepped thickness variation considered in [15]. A theoretical analysis is performed using Mindlin's two-dimensional (2D) plate equation [16] for TSh vibrations of a quartz plate.

\section{GOVERNING EQUATIONS}

Consider an AT-cut quartz plate, as shown in Fig. 1. The plate has a uniform thickness $2 h$ and a mass density $\rho$. There is a thin mass layer with a varying thickness on the top of the crystal plate. The density of the mass layer is $\rho^{\prime}$. Its varying thickness is $2 h^{\prime}$. The mass layer is assumed to be very thin. Only its inertia will be considered. Its stiffness will be neglected [1], [2]. Quartz is a material with very weak piezoelectric couplings. For a frequency analysis, we will neglect the small couplings as usual. In general, TSh vibration may be coupled to flexural motion. This coupling depends on the plate dimensions and is strong only for certain aspect (length/thickness) ratios of the plate [16]. For thin plates it is less likely to happen. For our purpose, it is sufficient to assume that the coupling to flexure has been avoided through 
design. Therefore, we consider TSh vibration with one displacement component $u_{1}\left(x_{1}, x_{2}, x_{3}, t\right)$ only.

Pure TSh modes are functions of the plate thickness coordinate $x_{2}$ and time $t$. The fundamental TSh mode is used most often in devices. It has a sinusoidal variation along the plate thickness with one nodal (zero) point at the plate middle plane. Higher order TSh modes have more nodal points along the plate thickness. For a plate with a nonuniform mass layer, these modes also have slow in-plane variations. In this paper we focus on the fundamental TSh mode.

Exact analysis of TSh modes with in-plane variations in anisotropic crystal plates using the three-dimensional equations of elasticity is mathematically very challenging and in most cases impossible. Mindlin proposed [16] to use simple and known variations along the plate thickness to approximate the modes and then obtain two-dimensional plate equations governing the in-plane variations of the modes. The small error due to the approximation can be minimized or removed using a correction factor. Mindlin's approach turned out to be very successful and many researchers followed. Specifically, for the most useful fundamental TSh mode we are considering, Mindlin used a linear variation to approximate the sinusoidal variation of the TSh displacement along the plate thickness, i.e.,

$$
u_{1}\left(x_{1}, x_{2}, x_{3}, t\right) \cong x_{2} \psi_{1}\left(x_{1}, x_{3}, t\right), \quad u_{2} \cong 0, \quad u_{3} \cong 0
$$

where $\psi_{1}\left(x_{1}, x_{3}, t\right)$ is a slowly varying function of the in-plane coordinates $x_{1}$ and $x_{3} . x_{2} \psi_{1}$ has one nodal point along the plate thickness when $x_{2}=0$ which is the plate middle plane. In (1), the variation along the plate thickness is already known. What is needed to determine $u_{1}$ is a two-dimensional plate equation governing the dependence of $\psi_{1}$ on $x_{1}$ and $x_{3}$. Such an equation is obtained by multiplying the corresponding three-dimensional equation of anisotropic elasticity by $x_{2}$ and integrating it through the plate thickness (moment operation) [16]. When a crystal plate carries a mass layer, the inertial effect of the mass layer can be brought into the plate equation using the traction boundary conditions at the plate top and/or bottom surface(s) and Newton's second law governing the motion of the mass layer [17], [18]. For the specific case of a quartz plate carrying a mass layer in free TSh vibration, the governing equation for $\psi_{1}$ is [17], [18]

$\gamma_{11} \frac{\partial^{2} \psi_{1}}{\partial x_{1}^{2}}+\gamma_{55} \frac{\partial^{2} \psi_{1}}{\partial x_{3}^{2}}-3 h^{-2} \kappa^{2} c_{66} \psi_{1}+(1+3 R) \rho \omega^{2} \psi_{1}=0$

where $\omega$ is the free vibration frequency which is to be determined and

$$
\begin{gathered}
\kappa^{2}=\frac{\pi^{2}}{12}(1+R), \quad R=\frac{\rho^{\prime} h^{\prime}}{\rho h}, \\
\gamma_{11}=\frac{s_{33}}{s_{11} s_{33}-s_{13}^{2}}, \quad \gamma_{55}=\frac{1}{s_{55}} .
\end{gathered}
$$

$s_{p q}$ are the usual elastic compliances. For AT-cut quartz, $\gamma_{11}=$ $79.27, \gamma_{55}=49.90 \times 10^{9} \mathrm{~N} / \mathrm{m}^{2} . R$ is the mass ratio between the mass layer and the crystal plate which is very small. We are interested in first-order effects of $R$ on resonant frequencies. $\kappa$ is the shear correction factor introduced by Mindlin. With the correction factor, the inaccuracy of resonant frequencies predicted by (2) is higher order infinitesimals of $R$. For small $R$, (2) can be approximately rewritten as

$$
\gamma_{11} \frac{\partial^{2} \psi_{1}}{\partial x_{1}^{2}}+\gamma_{55} \frac{\partial^{2} \psi_{1}}{\partial x_{3}^{2}}+\left[\rho \omega^{2}-\rho \omega_{\infty}^{2}(1-2 R)\right] \psi_{1}=0
$$

where

$$
\omega_{\infty}^{2}=\frac{\pi^{2} c_{66}}{4 h^{2} \rho} .
$$

$\omega_{\infty}$ is the frequency of the fundamental TSh mode of the crystal plate when the mass layer is not present. The difference between (2) and (5) is of the order of $\left(\omega^{2}-\omega_{\infty}^{2}\right) R$, which is a higher order infinitesimal because $\omega^{2}-\omega_{\infty}^{2}$ is of the order of $R$ and $R$ is very small. We consider the case when the mass layer thickness is varying according to

$$
2 h^{\prime}=2 h_{0}^{\prime}\left[1-f_{1}\left(x_{1}\right)-f_{3}\left(x_{3}\right)\right]
$$

where $2 h_{0}^{\prime}$ is the mass layer center thickness, $f_{1}$ and $f_{3}$ are slowly growing functions so that the mass layer is thick in the center and thin away from the center. Therefore, (7) can physically describe a nonuniform mass layer. The specific choice of the thickness variation in (7) is also for the mathematical convenience below so that the problem is solvable. Corresponding to (7), from $(3)_{2}$, we have

$$
R=R_{0}\left[1-f_{1}\left(x_{1}\right)-f_{3}\left(x_{3}\right)\right]
$$

where

$$
R_{0}=\frac{\rho^{\prime} h_{0}^{\prime}}{\rho h} .
$$

Substitution of (8) into (5) gives

$$
\begin{aligned}
\gamma_{11} \frac{\partial^{2} \psi_{1}}{\partial x_{1}^{2}}+ & \gamma_{55} \frac{\partial^{2} \psi_{1}}{\partial x_{3}^{2}}+ \\
& {\left[\rho \omega^{2}-\rho \omega_{0}^{2}-\rho \omega_{\infty}^{2} 2 R_{0}\left(f_{1}+f_{3}\right)\right] \psi_{1}=0 }
\end{aligned}
$$

where we have denoted

$$
\omega_{0}^{2}=\omega_{\infty}^{2}\left(1-2 R_{0}\right) .
$$

$\omega_{0}$ is the frequency of the fundamental TSh mode of a crystal plate with a mass layer of a uniform thickness $2 h_{0}^{\prime}$. We are interested in the so-called energy-trapped modes with the frequency $\omega$ within

$$
\omega_{0}<\omega<\omega_{\infty}
$$

These modes are large near the plate center and decay rapidly away from the center.

\section{FREE VIBRATION SOLUTION}

As a partial differential equation, (10) is separable. Let

$$
\psi_{1}\left(x_{1}, x_{3}\right)=F_{1}\left(x_{1}\right) F_{3}\left(x_{3}\right) .
$$




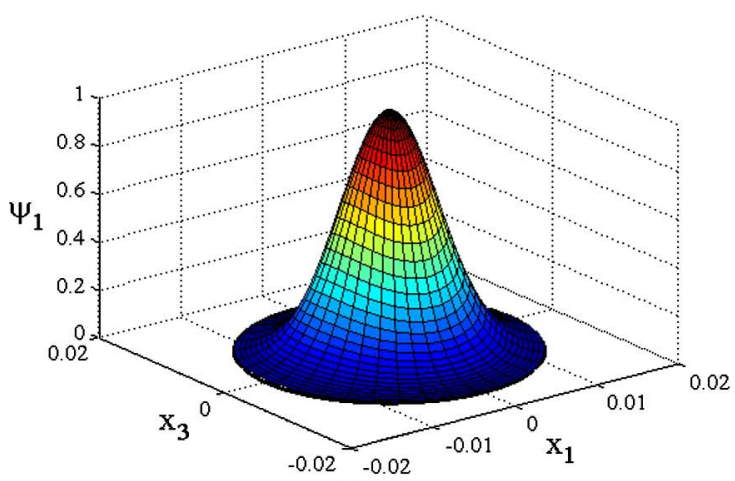

(a)

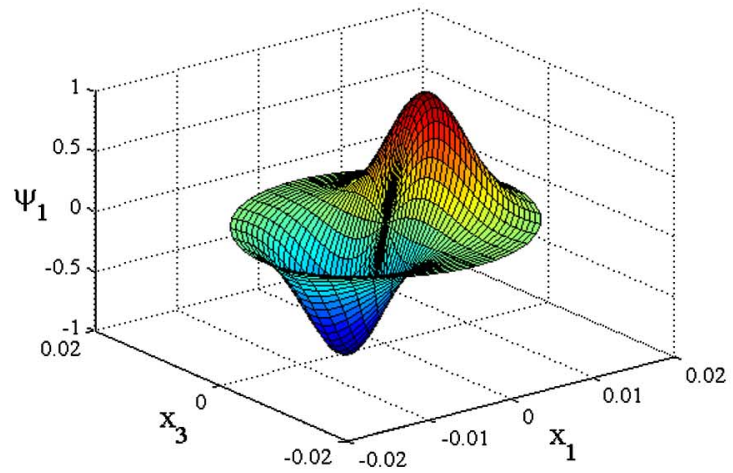

(c)

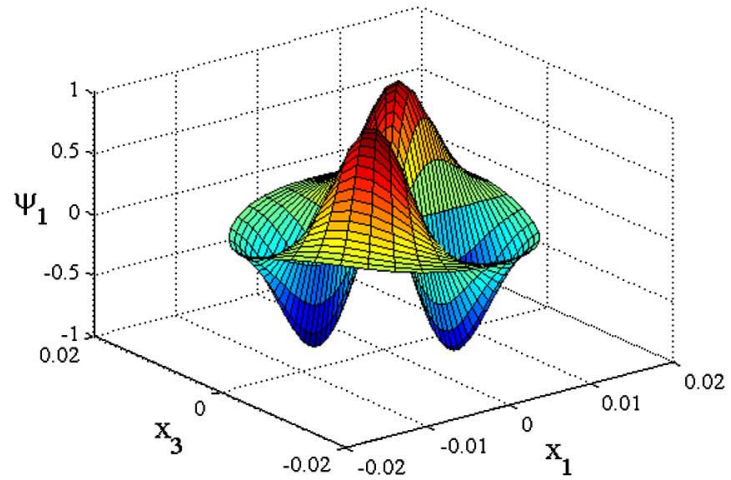

(e)

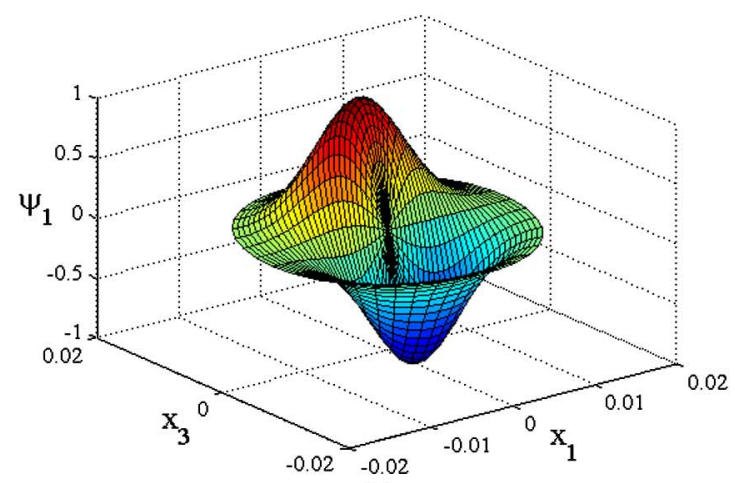

(b)

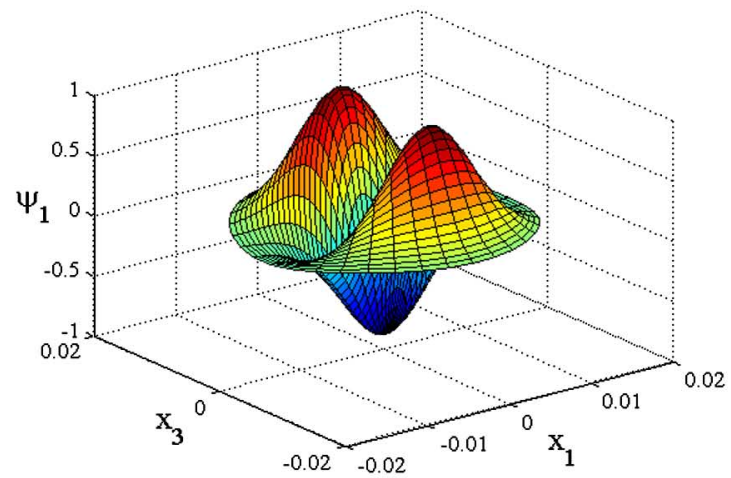

(d)

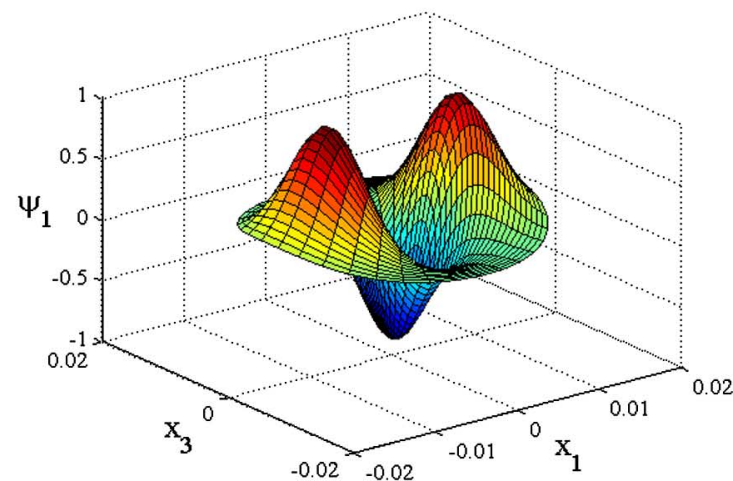

(f)

Fig. 2. Distribution of the TSh displacement of the first six trapped modes in the order of increasing frequency. (a) $\left(n_{1}, n_{3}\right)=(0,0)$. (b) $\left(n_{1}, n_{3}\right)=(0,1)$. (c) $\left(n_{1}, n_{3}\right)=(1,0)$. (d) $\left(n_{1}, n_{3}\right)=(0,2)$. (e) $\left(n_{1}, n_{3}\right)=(1,1)$. (f) $\left(n_{1}, n_{3}\right)=(2,0)$.

Then, using the standard method of separation of variables, from (10) we can obtain

$$
\begin{aligned}
& \gamma_{11} F_{1,11}+\rho \omega_{1}^{2} F_{1}-\rho \omega_{\infty}^{2} 2 R_{0} f_{1}\left(x_{1}\right) F_{1}=0 \\
& \gamma_{55} F_{3,33}+\rho \omega_{3}^{2} F_{3}-\rho \omega_{\infty}^{2} 2 R_{0} f_{3}\left(x_{3}\right) F_{3}=0
\end{aligned}
$$

where the separation constants $\omega_{1}$ and $\omega_{3}$ must satisfy the following equation from (10) which gives the resonant frequencies once $\omega_{1}$ and $\omega_{3}$ are determined

$$
\omega^{2}=\omega_{0}^{2}+\omega_{1}^{2}+\omega_{3}^{2}
$$

To be specific, we consider the case when

$$
f_{1}\left(x_{1}\right)=\beta_{1} x_{1}^{2}, \quad f_{3}\left(x_{3}\right)=\beta_{3} x_{3}^{2} .
$$

\section{Correspondingly}

$$
\begin{aligned}
& \gamma_{11} F_{1,11}+\rho \omega_{1}^{2} F_{1}-\rho \omega_{\infty}^{2} 2 R_{0} \beta_{1} x_{1}^{2} F_{1}=0 \\
& \gamma_{55} F_{3,33}+\rho \omega_{3}^{2} F_{3}-\rho \omega_{\infty}^{2} 2 R_{0} \beta_{3} x_{3}^{3} F_{3}=0 .
\end{aligned}
$$

Equations similar to (17) were also encountered in the study of contoured resonators with varying thicknesses [19]-[22]. Eq. (17) is mathematically analogous to Schrodinger's equation for a quantum mechanical harmonic oscillator [23]. The two equations in (17) have the same structure. Consider (17) first. Introduce the following change of variable:

$$
x_{1}=A_{1} \xi_{1} .
$$

Then, it can be verified that when

$$
A_{1}=\left(\frac{\gamma_{11}}{2 \rho \omega_{\infty}^{2} R_{0} \beta_{1}}\right)^{1 / 4}
$$




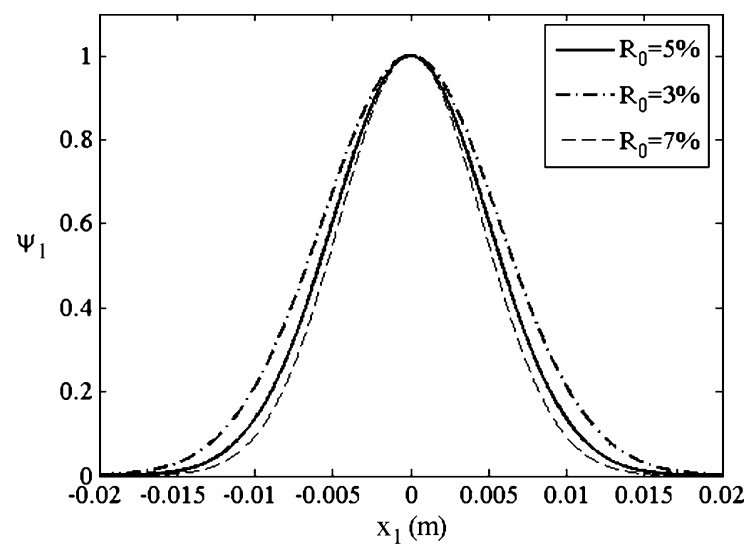

(a)

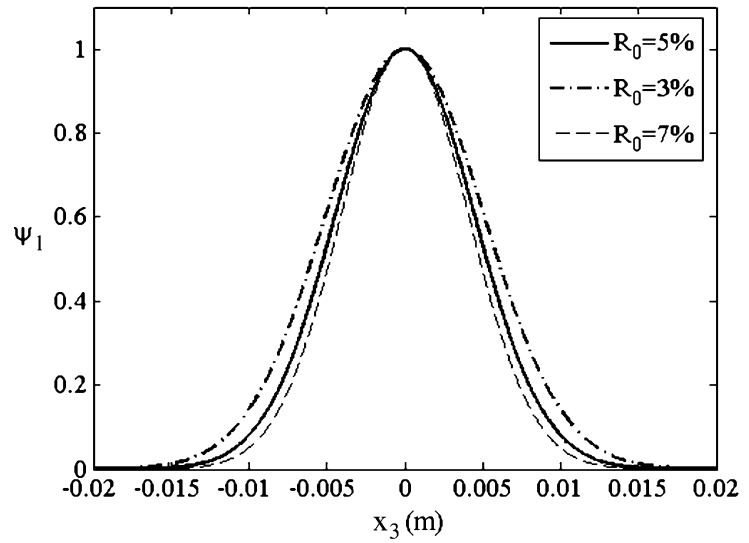

(b)

Fig. 3. Effects of $R_{0}$ on the first mode, $\beta=4444.4 \mathrm{~m}^{-2} \cdot f_{(0,0)}=1617327$, 1586981 , and $1555305 \mathrm{~Hz}$ when $R_{0}=3 \%, 5 \%$, and $7 \%$. (a) $x_{1}$ dependence. (b) $x_{3}$ dependence.

$(17)_{1}$ is transformed into

$$
\frac{d^{2} F_{1}\left(\xi_{1}\right)}{d \xi_{1}^{2}}+\left(\lambda_{1}-\xi_{1}^{2}\right) F_{1}\left(\xi_{1}\right)=0
$$

where

$$
\lambda_{1}=\rho \omega_{1}^{2} \frac{A_{1}^{2}}{\gamma_{11}} \quad \text { or } \quad \omega_{1}^{2}=\frac{\gamma_{11}}{\rho A_{1}^{2}} \lambda_{1} .
$$

(20) is the well-known Weber's equation [21], [22]. Let

$$
F_{1}\left(\xi_{1}\right)=\exp \left(-\xi_{1}^{2} / 2\right) H\left(\xi_{1}\right) .
$$

Eq. (20) becomes

$$
\frac{d^{2} H}{d \xi_{1}^{2}}-2 \xi_{1} \frac{d H}{d \xi_{1}}+\left(\lambda_{1}-1\right) H=0 .
$$

For odd integers of $\lambda$, the solution of (23) are the Hermite polynomials of different orders, i.e., [23]

$$
\lambda_{1}=2 n_{1}+1, \quad H\left(\xi_{1}\right)=H_{n_{1}}\left(\xi_{1}\right), \quad n_{1}=0,1,2,3 \cdots
$$

where [23], [24]

$$
\begin{aligned}
H_{0}= & 1 \\
H_{1}= & 2 \xi_{1} \\
H_{2}= & 4 \xi_{1}^{2}-2 \\
H_{3}= & 8 \xi_{1}^{3}-12 \xi_{1} \\
& \cdots .
\end{aligned}
$$

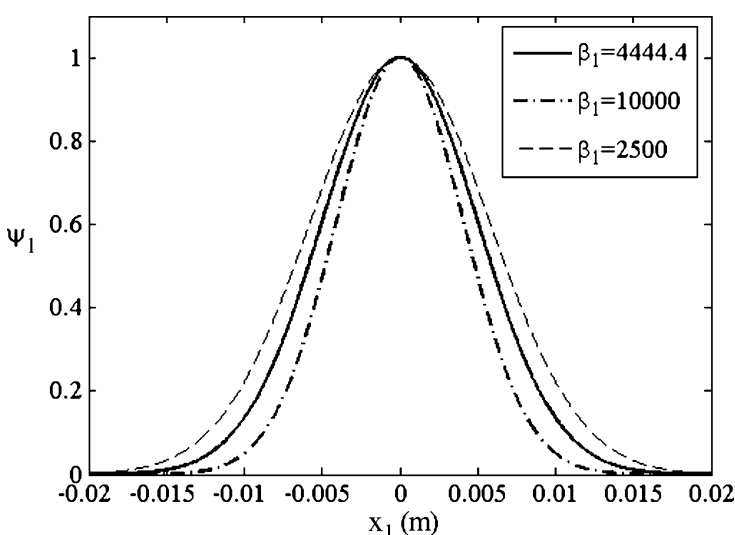

(a)

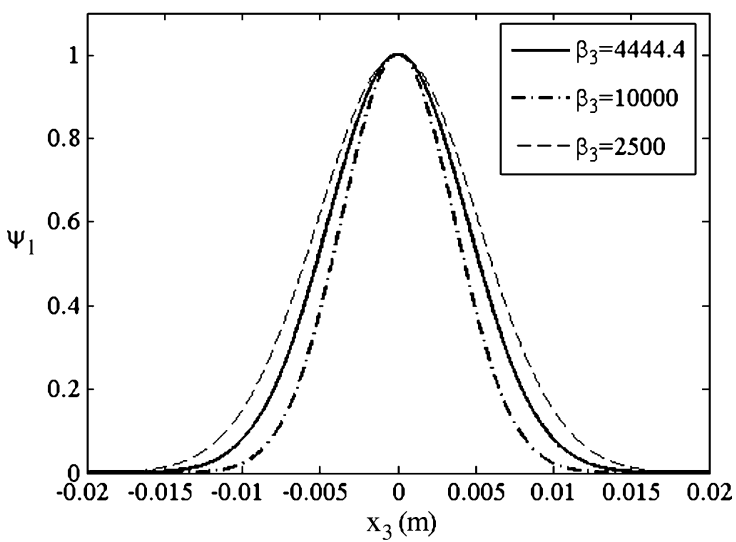

(b)

Fig. 4. Effects of $\beta$ on the first mode, $R_{0}=5 \% \cdot f_{(0,0)}=1582685$, 1586981 , and $1595538 \mathrm{~Hz}$ when $\beta=2500,4444.4$, and $10000 \mathrm{~m}^{-2}$. (a) $x_{1}$ dependence. (b) $x_{3}$ dependence.

Eq. (17) $)_{2}$ can be treated similarly. Substituting (11), (9), (6), $(21)_{2}$, and a similar equation from $(17)_{2}$ into (15) gives the resonant frequencies as

$$
\omega^{2}=\omega_{\infty}^{2}\left(1-2 R_{0}\right)+\frac{\gamma_{11}}{\rho A_{1}^{2}}\left(2 n_{1}+1\right)+\frac{\gamma_{55}}{\rho A_{3}^{2}}\left(2 n_{3}+1\right) .
$$

\section{NUMERICAL RESULTS}

As a numerical example, consider an AT-cut quartz resonator with $2 h=1 \mathrm{~mm}$, and $R_{0}=5 \%$. In real applications $R_{0}$ is usually less than $1 \%$. An exaggerated value of $R_{0}$ is chosen to show its numerical effects more clearly. In this case the TSh mode without a film has a frequency of $f_{\infty}=\omega_{\infty} /(2 \pi)=$ $1654638 \mathrm{~Hz}$. The frequency when the plate is with a uniform mass layer of $R_{0}=5 \%$ is $f_{0}=\omega_{0} /(2 \pi)=1569727 \mathrm{~Hz}$. $\beta_{1}=\beta_{3}=\beta=4444.4 \mathrm{~m}^{-2}$ is used. For this value of $\beta$, the film thickness decreases to zero when its radius is $15 \mathrm{~mm}$, a practically reasonable size. Calculations show that in this case the operating mode of $\left(n_{1}, n_{3}\right)=(0,0)$ in fact essentially decays to zero before it reaches the film boundary and thus does not really feel the film boundary.

Fig. 2 shows the first six modes in the interval of $\left(f_{0}, f_{\infty}\right)$ in the order of increasing frequency. $\left(n_{1}, n_{3}\right)=(0,0),(0,1),(1,0)$, $(0,2),(1,1)$, and $(2,0) . f_{(0,0)}=1586981, f_{(0,1)}=1602093$, $f_{(1,0)}=1606004, f_{(0,2)}=1617063, f_{(1,1)}=1620938$, $f_{(2,0)}=1624804 \mathrm{~Hz}$. The displacements of all these modes are 
essentially under the film. This is called energy trapping in TSh resonators. The modes are with different numbers of nodal lines in the $x_{1}$ and/or $x_{3}$ directions. Roughly, higher order modes have higher frequencies.

Figs. 3 and 4 show the effects of $R_{0}$ and $\beta$ on the most useful mode in devices with $\left(n_{1}, n_{3}\right)=(0,0)$. Considering the in-plane material anisotropy of the plate, both the mode variations along the $x_{1}$ and/or $x_{3}$ directions are shown separately. It is seen that when $R_{0}$ becomes larger or the film becomes thicker, the frequencies become lower and the modes are pushed toward the center (Fig. 3). When $\beta$ becomes larger, i.e., the film thickness variation is more rapid or the film becomes smaller, the frequencies become higher and modes are also pushed toward the center (Fig. 4).

\section{CONCLUSION}

When a thin mass layer is included into Mindlin's 2D plate equation for TSh modes of a quartz plate, the resulting equation is mathematically analogous to Schrodinger's equation for a particle in a potential field. Specifically, when the film thickness varies quadratically, the plate equation is the same as that of a quantum harmonic oscillator. There exist trapped modes whose motion is mainly under the film. When the film becomes thicker or smaller, the modes are pushed toward the center. In addition to resonant frequencies, impedance is another important property of a resonator-based sensor which needs to be obtained from an electrically forced vibration analysis and is beyond the model in this paper.

\section{REFERENCES}

[1] A. Ballato and T. J. Lukaszek, "Mass-loading of thickness-excited crystal resonators having arbitrary piezo-coupling," IEEE Trans. Sonics Ultrason., vol. SU-21, no. 4, pp. 269-274, Oct. 1974.

[2] J. A. Kosinski, "Thickness vibrations of flat piezoelectric plates with massy electrodes of unequal thickness," in Proc. IEEE Ultrasonics Symp., 2003, pp. 70-73.

[3] J. G. Miller and D. I. Bolef, "Acoustic wave analysis of the operation of quartz-crystal film-thickness monitors," J. Appl. Phys., vol. 39, no. 12, pp. 5815-5816, 1968.

[4] F. Boersma and E. C. van Ballegooyen, "Rotated y-cut quartz crystal with two different electrodes treated as a one-dimensional acoustic composite resonator," J. Acoust. Soc. Amer., vol. 62, no. 2, pp. 335-340, 1977.

[5] P. J. Cumpson and M. P. Seah, "The quartz crystal microbalance; radial/polar dependence of mass sensitivity both on and off the electrodes," Meas. Sci. Technol., vol. 1, no. 7, pp. 544-555, 1990.

[6] F. Josse, Y. Lee, S. J. Martin, and R. W. Cernosek, "Analysis of the radial dependence of mass sensitivity for modified-electrode quartz crystal resonators," Anal. Chem., vol. 70, no. 2, pp. 237-247, 1998.

[7] B. A. Martin and H. E. Hager, "Velocity profile on quartz crystals oscillating in liquids," J. Appl. Phys., vol. 65, no. 7, pp. 2630-2635, 1989.

[8] J. R. Vig and A. Ballato, "Comments on the effects of nonuniform mass loading on a quartz crystal microbalance," IEEE. Trans. Ultrason. Ferroelectr. Freq. Control, vol. 45, no. 5, pp. 1123-1124, 1998.

[9] C. van der Steen, F. Boersma, and E. C. van Ballegooyen, "The influence of mass loading outside the electrode area on the resonant frequencies of a quartz-crystal microbalance," J. Appl. Phys., vol. 48, no. 8, pp. 3201-3205, 1977.

[10] E. C. van Ballegooijen, F. Boersma, and C. van der Steen, "Influence of the thickness of tabs on the resonating properties of a quartz crystal," J. Acoust. Soc. Amer., vol. 62, no. 5, pp. 1189-1195, 1977.

[11] J. S. Yang, H. Xue, H. Y. Fang, Y. T. Hu, J. Wang, and L. J. Sheng, "Effects of electrodes with varying thickness on energy trapping in thickness-shear quartz resonators," IEEE. Trans. Ultrason. Ferroelectr. Freq. Control, vol. 54, no. 4, pp. 892-895, 2007.
[12] J. Wang, L. J. Shen, and J. S. Yang, "Effects of electrodes with continuously varying thickness on energy trapping in thickness-shear mode quartz resonators," ULTRASONIC, vol. 48, no. 2, pp. 150-154, 2008.

[13] J. S. Yang, Z. G. Chen, and H. P. Hu, "Electrically forced vibration of a thickness-twist mode piezoelectric resonator with non-uniform electrodes," Acta Mechanica Solida Sinica, vol. 20, no. 3, pp. 266-274, 2007.

[14] J. S. Yang, Z. G. Chen, and Y. T. Hu, "Vibration of a thickness-twist mode piezoelectric resonator with asymmetric, nonuniform electrodes," IEEE. Trans. Ultrason. Ferroelectr. Freq. Control, vol. 55, no. 4, pp. 841-848, 2008.

[15] N. Liu, J. S. Yang, and W. Q. Chen, "Effects of mass layer nonuniformity on a quartz crystal microbalance," IEEE Sensors J., DOI: 10.1109/ JSEN.2010.2064297, accepted for publication.

[16] R. D. Mindlin, "Thickness-Shear and flexural vibrations of crystal plates," J. Appl. Phys., vol. 22, no. 3, pp. 316-323, 1951.

[17] R. D. Mindlin and P. C. Y. Lee, "Thickness-shear and flexural vibrations of partially plated, crystal plates," Int. J. Solids Structures, vol. 2. no. 1, pp. 125-139, Jan. 1966.

[18] R. D. Mindlin, "High frequency vibrations of plated, crystal plates," in Progress in Applied Mechanics (the Prager Anniversary Volume). New York: Macmillan, 1963, pp. 73-84.

[19] C. J. Wilson, "Vibration modes of at-cut convex quartz resonators," $J$. Phys. D., vol. 7, no. 18, pp. 2449-2455, 1974.

[20] H. F. Tiersten and R. C. Smythe, "An analysis of contoured crystal resonators operating in overtones of coupled thickness shear and thickness twist," J. Acoust. Soc. Amer., vol. 65, no. 6, pp. 1455-1460, 1979.

[21] D. S. Stevens and H. F. Tiersten, "An analysis of doubly rotated quartz resonators utilizing essentially thickness modes with transverse variation," J. Acoust. Soc. Amer., vol. 79, no. 6, pp. 1811-1826, 1986.

[22] H. F. Tiersten, B. J. Lwo, and B. Dulmet, "Transversely varying thickness modes in trapped energy resonators with shallow and beveled contours," J. Appl. Phys., vol. 80, no. 2, pp. 1037-1046, 1996.

[23] R. H. Dicke and J. P. Wittke, Introduction to Quantum Mechanics, 1st ed. Reading, MA: Addison Wesley, 1961.

[24] L. C. Andrews, Special Functions of Mathematics For Engineers, 2nd ed. Oxford, U.K.: Oxford Univ. Press, 1998.

Nan Liu is currently working towards the Ph.D. degree at University of Nebraska-Lincoln, Lincoln.

Jiashi Yang received the B.E. and M.E. degrees in engineering mechanics from Tsinghua University, Beijing, China, in 1982 and 1985, respectively, and the $\mathrm{Ph} . \mathrm{D}$. degree in civil engineering from Princeton University, Princeton, NJ, in 1994.

He was a Postdoctoral Fellow from 1993 to 1994 at the University of Missouri-Rolla, Rolla, and from 1994 to 1995 at Rensselaer Polytechnic Institute, Troy, NY. He was employed by Motorola, Inc., Schaumburg, IL, from 1995 to 1997. Since 1997, he has been an Assistant and Associate Professor at the Department of Engineering Mechanics, University of Nebraska-Lincoln, Lincoln. He has published over 180 journal papers on electromechanical materials and devices, and three books: An Introduction to the Theory of Piezoelectricity, The Mechanics of Piezoelectric Structures, and Analysis of Piezoelectric Devices.

Weiqiu Chen received the B.S. degree in engineering mechanics and the Ph.D. degree in solid mechanics from Zhejiang University, Hangzhou, China, in 1990 and 1996, respectively.

From 1997 to 1999, he was a Postdoctoral Fellow at The University of Tokyo, Tokyo, Japan. Since 1996, he has been working in the Department of Civil Engineering, Zhejiang University, where he was promoted to be an Associate Professor and Full Professor in 1999 and 2000, respectively. He is now a Full Professor and Vice Director of the Institute of Applied Mechanics, Zhejiang University. He has coauthored three monographs, two in English and one in Chinese. He has published nearly 200 journal papers, among which about 150 are in international journals. His research interests include mechanics of piezoelectric materials and devices, vibration and waves in structures, and analytical methods in applied mechanics. 